\title{
ROOT TIP MIGRATION INTO THE INFUNDIBULUM OF THE MAXILLARY SINUS AFTER COMPLICATED FIRST MOLAR EXTRACTION
}

\author{
Isabel Miclotte ${ }^{1,2 a}{ }^{\mathbb{B}}$, Laurence Verstraete ${ }^{1,2 a}{ }^{(\mathbb{D})}$, Constantinus Politis ${ }^{1,2 b}$ \\ 1OMFS-IMPATH Research Group, Department of Imaging and Pathology, KU Leuven, Leuven, Belgium \\ 2Department of Oral and Maxillofacial Surgery, University Hospitals Leuven, Leuven, Belgium
}

${ }^{\mathrm{a} M D, D D S}$

${ }^{b} M D, D D S$, MHA, MM, PhD, Professor, Head

Aim: To show how an unsuccessful retrieval attempt of a broken root tip of an upper molar could lead to dislocation into the maxillary sinus and and end up at the ostium of the maxillary sinus, necessitating a FESS procedure for retrieval.

Summary: An upper right first molar was removed with forcesp extraction. The extraction was complicated with a root fracture. Manipulating the residual root tip resulted in root tip dislocation into the maxillary sinus. It was decided to leave the root tip at the bottom of the sinus, but in the following months the root tip migrated and got stuck into the ostium of the maxillary sinus. There, it was retrieved using functional endoscopic sinus surgery (FESS).

Key Learning Points: 1. Manipulating a residual root tip after forceps extraction of an upper molar significantly increases the risk of root tip dislocation into the maxillary sinus. 2. Broken root tips of upper molar roots protruding into the maxillary sinus need not to be removed when it concerns healthy vital pulps. 3. Retrieval attempts of broken root tips can lead to dislocation of the root tip into the maxillary sinus if the roots were protruding into the maxillary sinus or if an insufficient bony barrier separates the maxillary sinus from the alveolar socket. 4 . Cone beam ct is the radiological exam of preference to locate a dislocated root tip in the upper jaw. 5. A root tip stuck at the ostium of the maxillary sinus is best removed with a FESS-procedure.

Keywords: Tooth extraction; Root tip fracture; Functional endoscopic sinus sugery.

OPEN ACCESS This is an
Open Access article under the CC
BY-NC 4.0 license.
Citeer-Reviewed Article
Citation: Miclotte I,Verstraete L,
Politis C. Root tip migration into the
infundibulum of the maxillary sinus after
complicated first molar extraction Stoma
Edu J.2019;6(2):125-128
Received: May 06, 2019
Revised: May 22, 2019
Accepted: June 14, 2019
Published: June 18, 2019
*Corresponding author:
Prof. Dr. Constantinus Politis
Department of Oral and Maxillofacial
Surgery, University Hospitals Leuven,
Leuven, Belgium
Kapucijnenvoer 33, 3000 Leuven,
Belgium
Tel: / Fax: 0032 (0)163332462,
e-mail: constantinus.politis@uzleuven.be
Copyright: @ 2019
the Editorial Council for the
Stomatology Edu Journal.

\section{Introduction}

Extraction of maxillary molars with multiple and separated roots carries a risk of root fracture. Moreover, fractured roots can be displaced into the maxillary sinus, potentially causing oroantral fistula, sinusitis, cellulitis, and subdural empyema [1]. Root displacement into the maxillary sinus can be detected by imaging, which also provides information regarding the root size and location within the sinus.

Some studies suggest that displaced root fragments should be left in the sinus. However, due to the potential development of complications, surgical removal of displaced root tips should always be considered. There are three major methods of removing root tips displaced into the maxillary sinus: a crestal approach, a bone window approach, and a more recently described endoscopic approach [2]. Here we report the case of a displaced root fragment from a first upper molar on the right side, which was located using cone-beam $C T$ imaging (CBCT). Applying an endoscopic approach, the fragment was removed six months after the extraction, after mucosal healing, and in the absence of sinusitis.

\section{Case history}

A 14-year-old female was referred by the orthodontic department for extraction of the four first molars (16, 26, 36, and 46) for orthodontic reasons (Fig. 1). Due to the presence of deep restorations, it was decided to remove the first molars rather than remove four premolars. The deep restorations on teeth 46 and 36 were visible on a panoramic radiograph (Fig. 2). CBCT

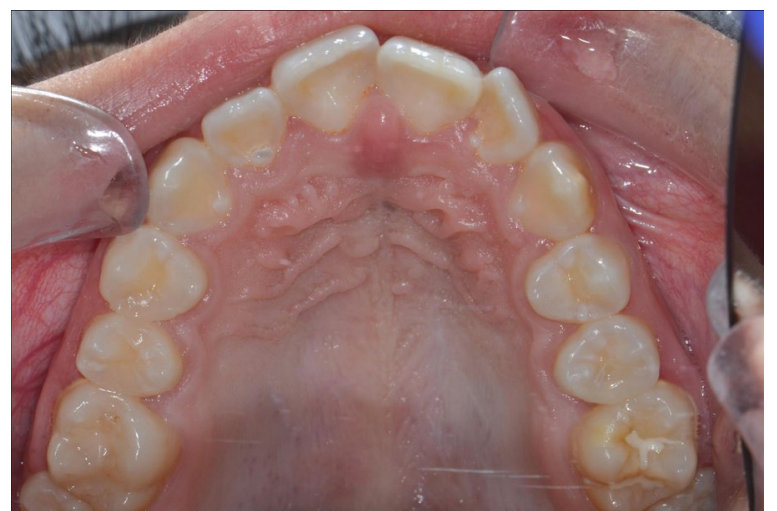

Figure 1. Intra-oral picture of the upper teeth, showing a restored first upper right molar. 


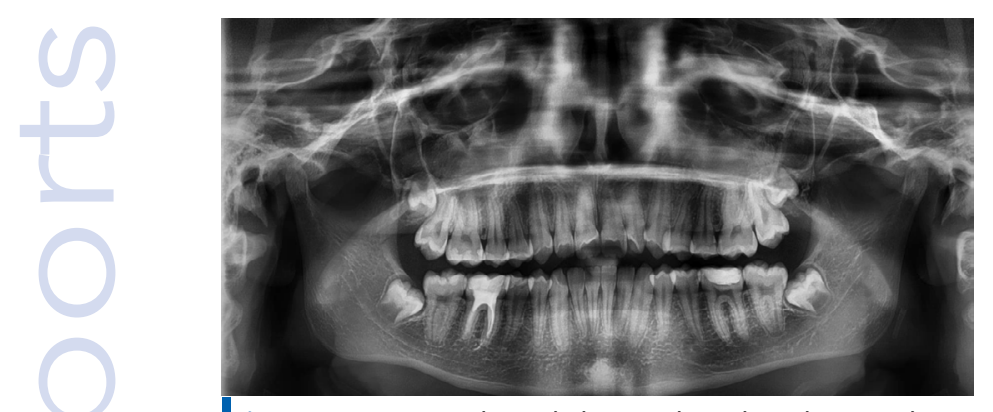

Figure 2. Panoramic radiograph showing close relation between the upper molars and the maxillary sinus.

was not performed prior to extraction. The molars were removed using forceps extraction under local anesthesia, one at a time, in four sessions. The local anesthetic used was articaine $4 \%$ with 1:200,000 epinephrine (Septodont, France). The extractions of elements 26, 46, and 36 were uneventful. However, upon forceps extraction of element 16, the distovestibular root tip was fractured. Efforts were made to remove the displaced root tip using an apical pick instrument, and then with root tip removal forceps. However, the root tip disappeared cranially and could not be retrieved. The root tip was not detected by transalveolar endoscopy or apical X-ray (Fig. 3). CBCT was immediately performed, which revealed the root tip at the base of the maxillary sinus (Fig. 4). This position suggested perforation of the sinus membrane; therefore, the alveolus was filled with four leukocyte-rich and platelet-rich fibrin (L-PRF) membranes. It was decided not to make another immediate attempt to remove the lost root tip, but rather to observe the situation and to reevaluate after four months. Healing was uneventful. Four months later, a new CBCT revealed that the displaced root tip had migrated towards the ostium of the right maxillary sinus (Fig. 5). Despite this position, the maxillary sinus showed no opacification, no mucosal thickening was visualized on CBCT, and the patients were asymptomatic. Two months later, a new CBCT was performed, showing the root tip at the same position. It was then decided to remove the root tip to prevent future complications. Believing that a Caldwell-Luc approach would be too traumatic, a functional endoscopic sinus surgery (FESS) procedure was proposed. The FESS procedure was performed under general anesthesia at the ear, nose, and throat (ENT) department. The root tip was retrieved. Further healing was uneventful. After healing of the four extraction wounds with an uneventful course, orthodontic therapy was immediately restarted.

\section{Discussion}

Dental extractions are a common oral procedure performed in dental and maxillofacial departments. The upper molar removal is sometimes accompanied by root fracture or oro-antral communication. In a series of 2355 dental extractions, the incidence of oro-antral communication was $0.56 \%$ after $\mathrm{M} 3$

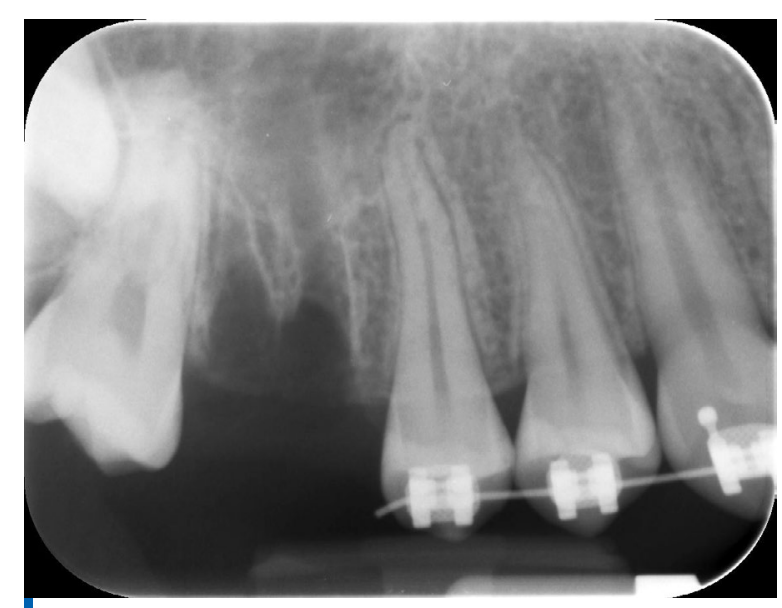

Figure 3. Intra-oral picture of the upper teeth, showing a restored first upper right molar.

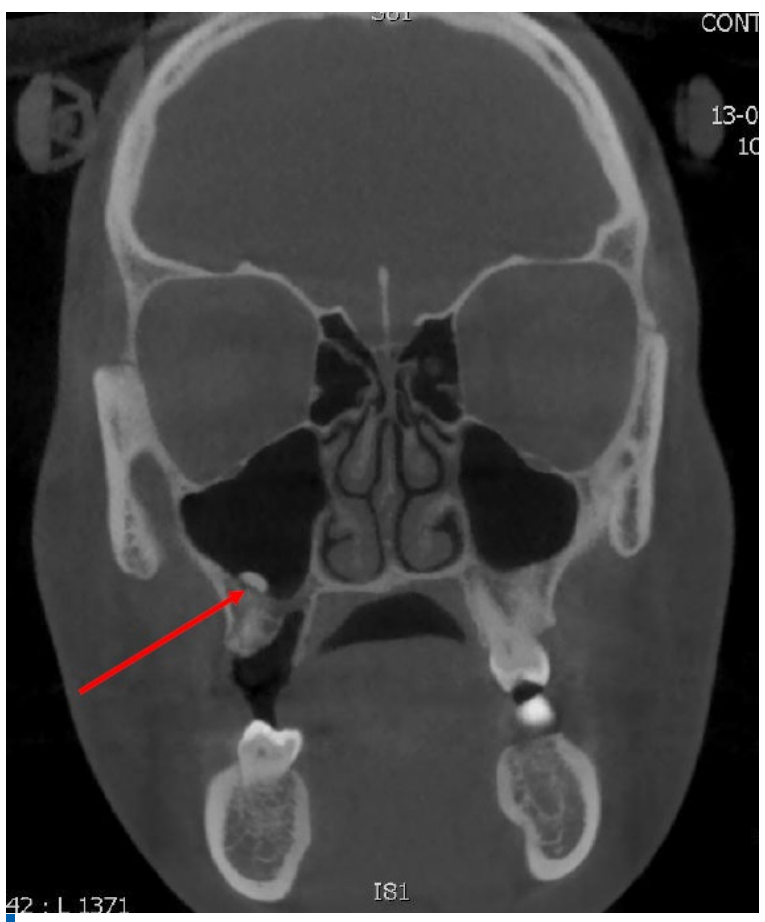

Figure 4. Panoramic radiograph showing close relation between the upper molars and the maxillary sinus.

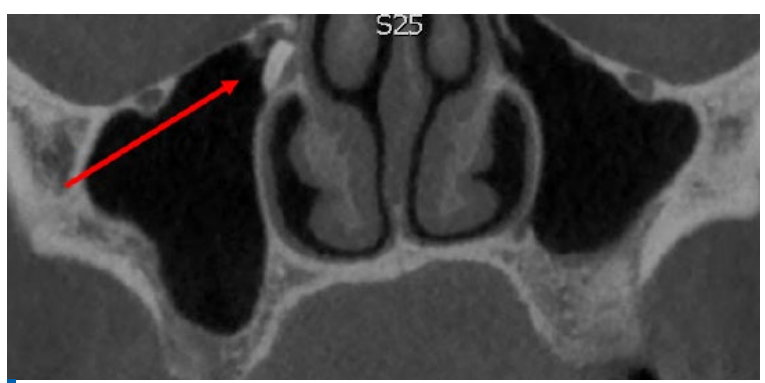

Figure 5. Intra-oral picture of the upper teeth, showing a restored first upper right molar.

extractions and $0.94 \%$ after non-M3 extractions [3]. Rarely, a fractured root migrates into the maxillary sinus. Such displacement depends on several factors, including the surgical technique (elevating roots with excessive force) and the bony interface between the alveolar socket and the sinus membrane. In a series of 1596 maxillary third molar extractions, only one case of tooth or root fragment displacement into 
the maxillary sinus was reported [4]. In another large series, no root tip migration was reported [3].

When a displaced root fragment is detected before alveolar socket healing, a crestal approach can be attempted to remove the fragment. This method involves enlargement of the socket to visualize the fragment or to rinse the maxillary sinus with saline. The disadvantages of this technique include damage to the alveolar bone, which may negatively influence the healing procedure and the residual bone height [2]. Some studies suggest leaving the root fragment in the sinus if it is below $3 \mathrm{~mm}$ in size, and if there is no evidence of sinusitis or other local disease [1]. When a displaced root fragment is diagnosed after mucosal healing, fragment retrieval can be performed using a bone window approach or an endoscopic approach. The traditional method is a Caldwell-Luc (bone window) approach in which fenestration of the anterior wall of the maxillary sinus provides a clear vision and improves the surgical range. However, this procedure is associated with complications, such as severe reactive swelling and nerve damage [2].

Alternatively, endoscopically-assisted procedures are reliable and minimally invasive methods for removing root fragments displaced into the maxillary sinus. Access for an endoscopic procedure can be obtained through the anterior maxillary sinus wall, or through the middle or inferior nasal meatus (FESS) [2]. This technique provides direct visibility of the surgical area and minimal risks of bleeding

\section{References}

1. Huang IY, Chen CM, Chuang FH. Caldwell-Luc procedure for retrieval of displaced root in the maxillary sinus. Oral Surg Oral Med Oral Pathol Oral Radiol Endod. 2011;112(6):e59-e63. [Full text links] [CrossRef] [PubMed] Google Scholar Scopus

2. Wang X, Li S. Removal of the residual roots displaced into the maxillary sinus via endoscopy. J Craniofac Surg. 2018;29(4):10341036.

[Full text links] [CrossRef] [PubMed] Google Scholar Scopus

3. Miclotte I, Agbaje JO, Spaey Y, et al. Incidence and treatment of complications in patients who had third molars or other teeth extracted. Br J Oral Maxillofac Surg. 2018;56(5):388-393.

[Full text links] [CrossRef] [PubMed] Google Scholar Scopus and nerve injury. However, endoscopic procedures require general anesthesia and their success depends on the operator experience.

In the presently reported case, immediate retrieval of the root tip displaced in the maxillary sinus was attempted, but without success. Although the fragment was large, immediate performance of a Caldwell-Luc procedure was considered too invasive in this young patient. After six months of follow-up with repeated $\mathrm{CBCT}$ imaging, it was deemed necessary to remove the fragment to avoid future complications. Instead of the Caldwell-Luc procedure, the less invasive FESS approach was performed, with a successful outcome.

\section{Conclusions}

Manipulating a residual root tip after forceps extraction of an upper molar-when little bony interface exists between the alveolar socket and the sinus membrane-significantly increases the risk of root tip dislocation into the maxillary sinus. Migration towards the ostium of the maxillary sinus is rare but possible. A FESS procedure is an efficient approach to retrieving the root tip from the maxillary sinus ostium, while keeping sinus function intact.

\section{Author contributions}

All authors contributed to reporting the case, performing the literature review, and drafting the manuscript.

4. Rothamel D, Wahl G, d'Hoedt B, et al. Incidence and predictive factors for perforation of the maxillary antrum in operations to remove upper wisdom teeth: prospective multicentre study. $\mathrm{Br}$ J Oral Maxillofac Surg. 2007;45(5):387-391.

[Full text links] [CrossRef] [PubMed] Google Scholar Scopus

\section{Isabel MICLOTTE

MD, DDS
OMFS-IMPATH Research Group
Department of Imaging and Pathology, KU Leuven
Leuven, Belgium

Dr. Isabel Miclotte was born in 1986 and was recognised as an OMFS surgeon in 2019. She will become senior resident of the department of Oral and Maxillo Facial Surgery at Leuven University Hospitals as of November 2019. Dr. Miclotte has published on complications of extractions and has elaborated guidelines for dento-alveolar surgery in patients under anticoagulant therapy. Her clinical focus is in orthodontic and orthognathic surgery. 


\section{Questions}

1. Which is the best radiographic exam to find a dislocated roottip of an upper molar tooth?

口a. Apical radiograph;

b. Panoramic radiograph;

口c. MRI;

$\square$ d. Cone-beam CT scan.

2. Which root is most often involved in an oro-antral communication?

口a. Third molar;

ab. Second molar;

ac. First molar;

ad. First premolar.

3. Which is the best technique to retrieve a dislocated root tip of an upper molar tooth at the ostium of the maxillary sinus?

$\square$ a. Denker technique;

b. Caldwell Luc technique;

ac. FESS technique;

ad. Rehrmann flap.

4. Which precaution could be taken to avoid dislocation of a root tip into the maxillary sinus?

$\square$ a. Leaving a broken roottip undistrubed;

$\square$ b. Frequent peroperative repeats of the Valsalva manoeuver;

uc. Performing the extraction in a supine position;

ud. Performing the extraction under general anaesthesia. 